\title{
Design of Multilevel Sequential Rotation Feeding Networks Used for Circularly Polarized Microstrip Antenna Arrays
}

\author{
Aixin Chen, ${ }^{1}$ Chuo Yang, ${ }^{1}$ Zhizhang Chen, ${ }^{2}$ Yanjun Zhang, ${ }^{1}$ and Yingyi $\mathrm{He}^{3}$ \\ ${ }^{1}$ School of Electronic and Information Engineering, Beihang University, Beijing 100191, China \\ ${ }^{2}$ Department of Electrical and Computer Engineering, Dalhousie University, Halifax, NS, Canada B3J $2 X 4$ \\ ${ }^{3}$ Institute of Electronic Engineering, China Academy of Engineering Physics, Mianyang, Sichuan 621900, China \\ Correspondence should be addressed to Aixin Chen, axchen@buaa.edu.cn
}

Received 8 March 2012; Accepted 11 July 2012

Academic Editor: Hala A. Elsadek

Copyright ( 2012 Aixin Chen et al. This is an open access article distributed under the Creative Commons Attribution License, which permits unrestricted use, distribution, and reproduction in any medium, provided the original work is properly cited.

\begin{abstract}
Sequential rotation feeding networks can significantly improve performance of the circularly polarized microstrip antenna array. In this paper, single, double, and multiple series-parallel sequential rotation feeding networks are examined. Compared with conventional parallel feeding structures, these multilevel feeding techniques present reduction of loss, increase of bandwidth, and improvement of radiation pattern and polarization purity. By using corner-truncated square patch as the array element and adopting appropriate level of sequential rotation series-parallel feeding structures as feeding networks, microstrip arrays can generate excellent circular polarization $(\mathrm{CP})$ over a relatively wide frequency band. They can find wide applications in phased array radar and satellite communication systems.
\end{abstract}

\section{Introduction}

Circularly polarized (CP) microstrip antenna array is more often used in wireless communication systems as they can be easily mounted on mobile devices and provide more focused radiation beams for better weather penetration and mitigation of multipath reflections [1]. Generally, CP patch antennas can be designed by using single-feed and dual-feed configurations. Both configurations can excite orthogonal modes with a quadrature phase shift $[2,3]$. For instance, in [2], by using a simple feed structure and a cornertruncated square patch with a thick air-layer substrate, a single-element antenna can have a $\mathrm{CP}$ bandwidth larger than $10 \%$. In [3], dual-feed configuration and sequential rotation feeding technique are applied to achieve a $30 \% \mathrm{CP}$ bandwidth.

However, problems and issues still exist with a CP array in particular related to the feed network, such as ohmic and dielectric losses, parasitic radiation, and the excitation of surface waves in dielectric substrates. Especially, in $\mathrm{Ka}$ band or at millimeter-wave frequency $[4,5]$, the problems become more severe and have more serious adverse effects on gain and impedance bandwidth than those in lower frequency bands. One way to improve the performance is to increase size. Another way is to develop efficient feed network configuration [6-10]. In [6], a microstrip antenna array with a relative gain bandwidth of $4.4 \%$ with less than $1 \mathrm{~dB}$ of gain ripple is developed with the use of a low-loss parallel-series feed topology. The work in [8] proposes a novel $2 \times 2$ patch antenna array with a serial feed arrangement which can be easily employed as subarrays in larger arrays.

In this paper, we propose single, double, and multiple series-parallel sequential rotation feeding networks in $\mathrm{Ka}$ band for use with 4-element [11], 64-element [12], and 256element CP microstrip antenna arrays, respectively. In order to show effectiveness of these multilevel feeding networks, they are compared with the conventional parallel feeding networks.

This paper is organized in the following manner. First, the structures of single, double and multiple series-parallel sequential rotation feeding networks and their comparisons with the conventional parallel feeding networks are presented in Section 2. Then, in Section 3, the simulated and/or measured results of the arrays with the proposed feeding networks are shown, compared, and discussed. Finally, in Section 4 , the conclusion is drawn. 


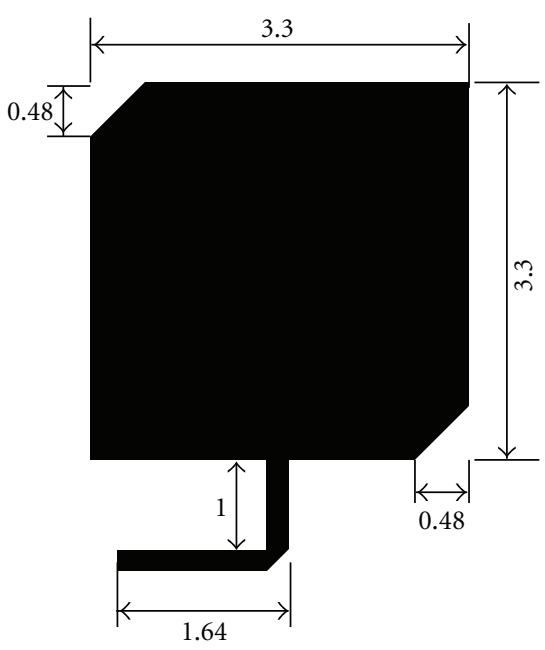

(a)

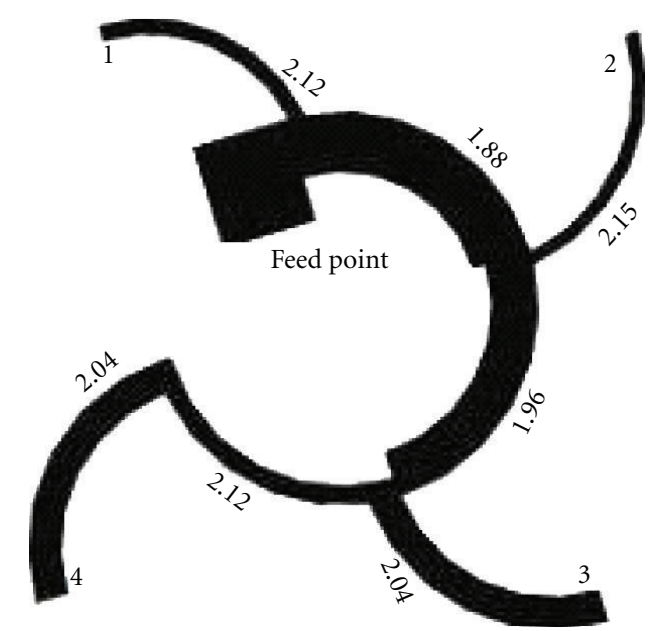

(b)

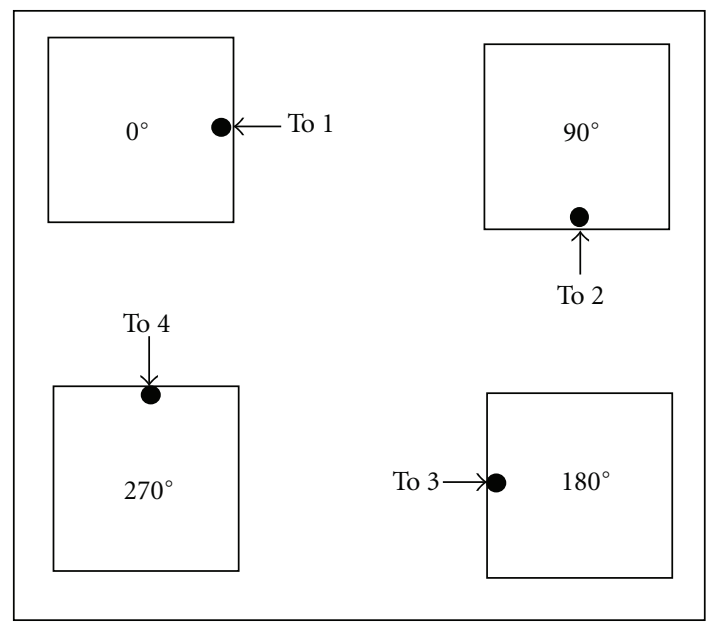

(c)

FIGURE 1: (a) Configuration of the single patch element, (b) the proposed single series-parallel sequential rotation feeding network, and (c) four patch elements (unit: millimeters).

\section{The Proposed Feeding Networks and Antenna Arrays}

Figure 1 shows the configuration of the single patch element, the proposed single series-parallel sequential rotation feeding network and the way four patch elements is connected to the feeding network. These four elements are arranged in a $2 \times 2$ square position with element angular orientation and feed phase arranged in a $0^{\circ}, 90^{\circ}, 180^{\circ}$, and $270^{\circ}$ fashion. The phase arrangement, as well as power distribution, is achieved by the feed lines of quarter-wavelength transformers and power dividers in the feeding network. The series-parallel sequential rotation feeding network and the four radiating elements together can form a 4-element CP antenna array. The reason that a sequential rotation feeding network can significantly improve the performance of circularly polarized antenna arrays is primarily attributed to its capability of phase arrangement and equal power split.
The array element is a corner-truncated microstrip patch $[11,12]$. The dimensions of the patch element and the feeding network are shown in Figures 1(a) and 1(b), respectively. With the application of single series-parallel sequential rotation feeding network, the 4-element CP microstrip antenna array can be obtained. Taking 4-element microstrip antenna array as a subarray, and applying double and multiple sequential rotation feeding networks, respectively, the 64-element and 256-element CP antenna arrays can then be obtained. In all cases, the spacing between adjacent elements is $0.77 \lambda$, where $\lambda$ is the free-space wavelength of the central frequency of $29 \mathrm{GHz}$. The substrate material is $\mathrm{RT} /$ duriod5880. It has a thickness of $0.254 \mathrm{~mm}$ with a relative dielectric constant of 2.2 and a dielectric loss tangent of 0.0009 .

Figure 2 shows the final layout of the arrays. Figure 2(a) is with the single series-parallel sequential rotation feeding network. Figure 2(b) is the conventional parallel feeding 


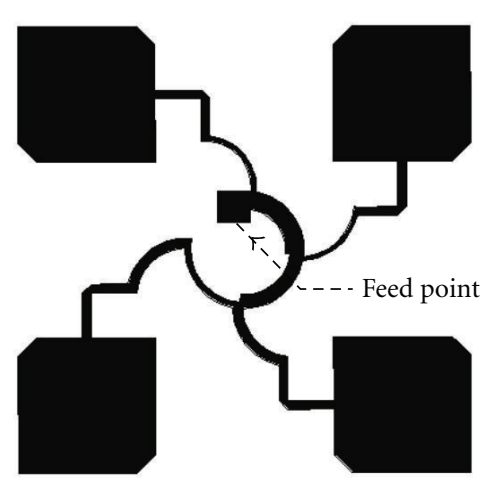

(a)

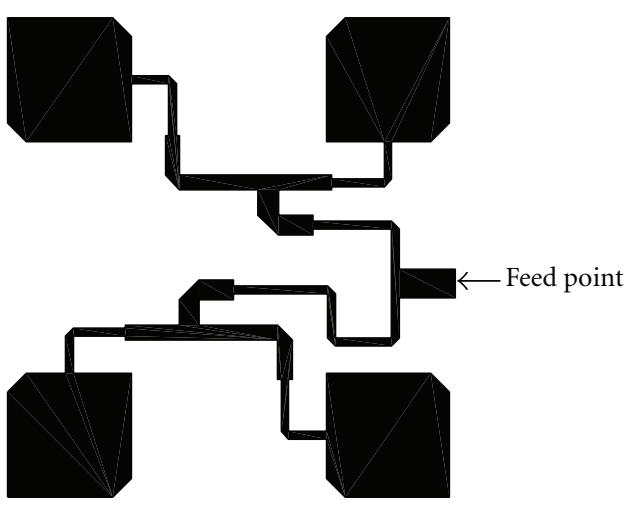

(b)

FIGURE 2: 4-element CP antenna arrays; (a) with the single series-parallel sequential rotation feeding network; (b) with the conventional parallel feeding network.

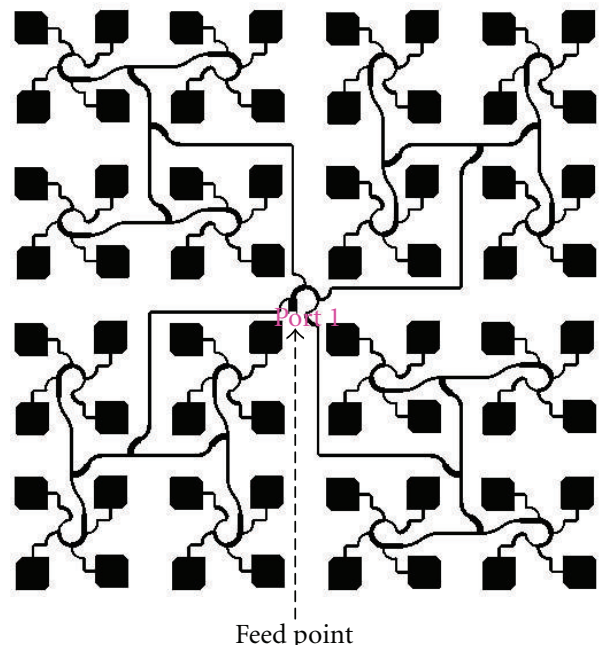

(a)
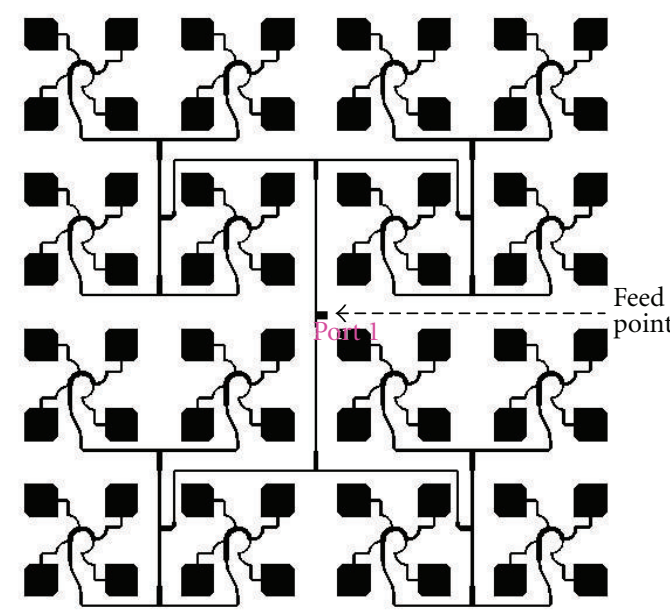

(b)

FIGURE 3: 64-element CP antenna arrays; (a) with the proposed double series-parallel sequential rotation feeding network; (b) with the conventional parallel feeding network.

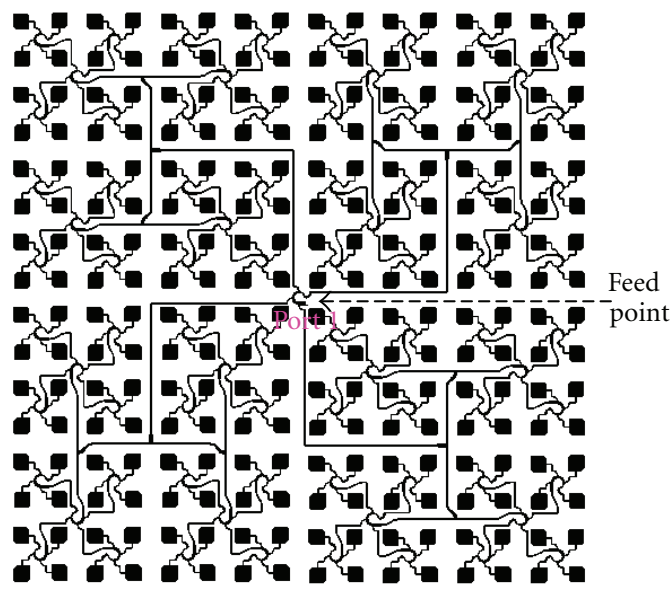

(a)

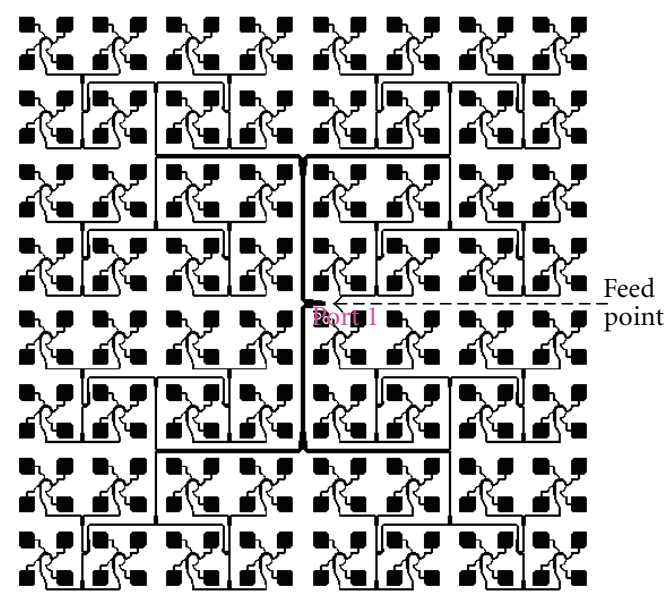

(b)

Figure 4: 256-element CP antenna arrays; (a) with the proposed multiple series-parallel sequential rotation feeding network; (b) with the conventional parallel feeding network. 


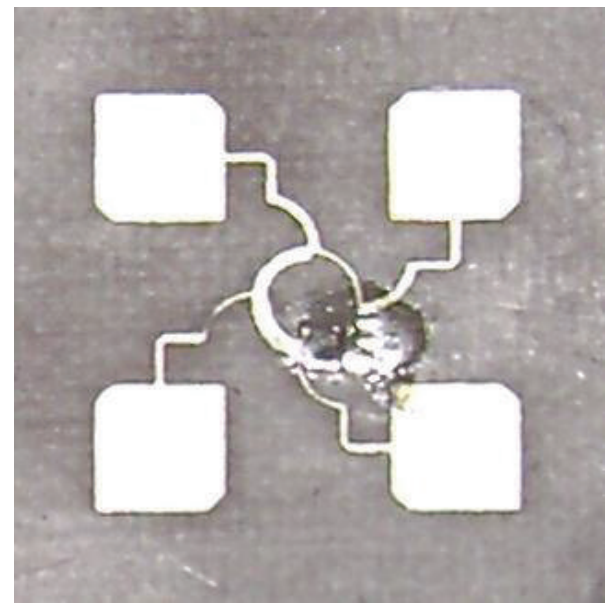

FIgURE 5: Photograph of 4-element CP antenna array with the proposed single sequential rotation feeding network [11].

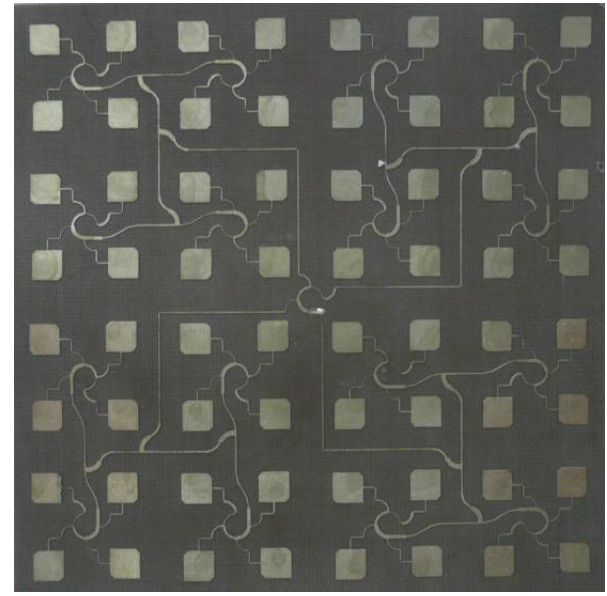

FIGURE 6: Photograph of 64-element CP antenna array with the proposed double sequential rotation feeding network [12].

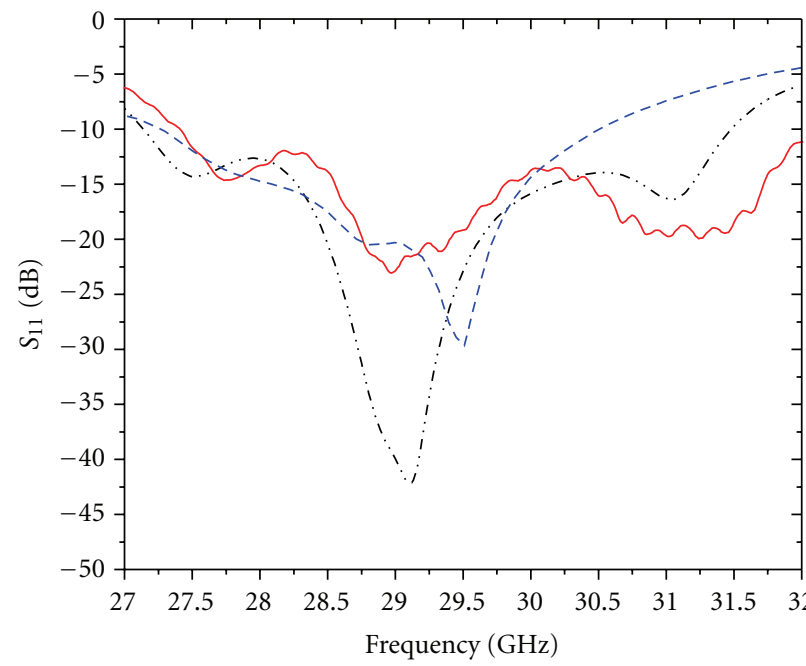

. - ... Simulated result of the proposed 4-element CP antenna array

Measured result of the proposed 4-element CP antenna array

- - - Simulated result of the conventional 4-element CP antenna array

Figure 7: Measured and simulated $S_{11}$ of the 4 -element CP antenna arrays. 


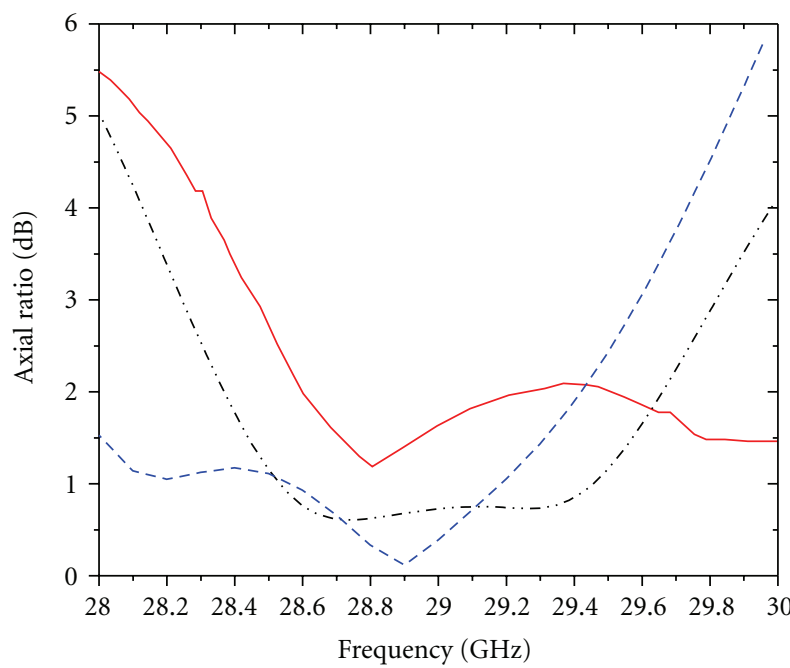

. - ... Simulated result of the proposed 4-element $\mathrm{CP}$ antenna array

- Measured result of the proposed 4-element CP antenna array

- - Simulated result of the conventional 4-element CP antenna array

Figure 8: Measured and simulated axial ratio of the 4-element CP antenna arrays.

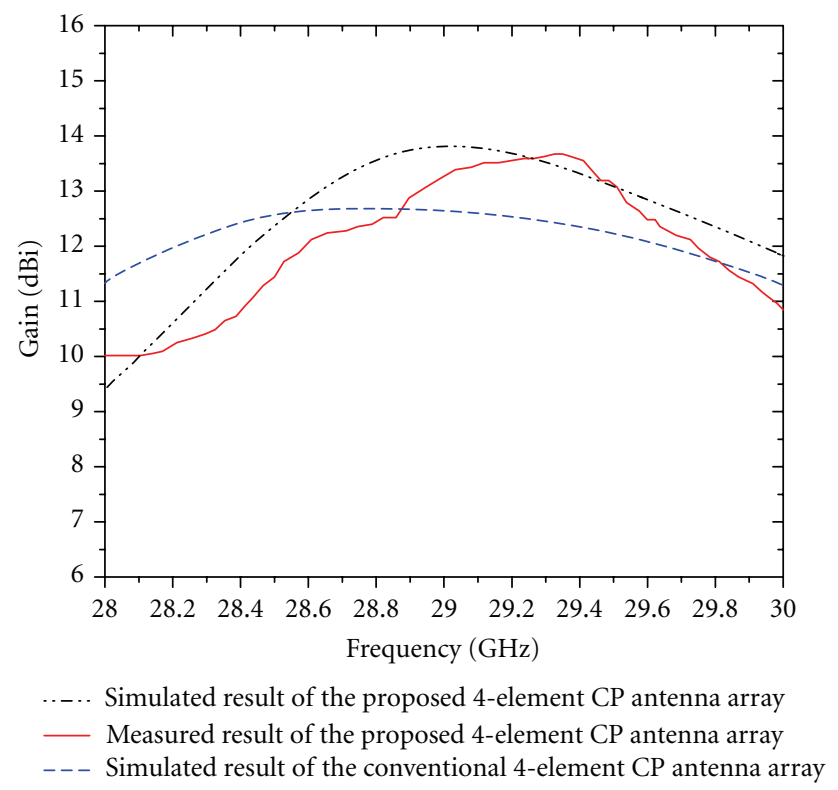

FIGURE 9: Measured and simulated gain of the 4-element CP antenna arrays.

network for the comparison and reference purpose. The phase shift with the reference array is also arranged in a $0^{\circ}$, $90^{\circ}, 180^{\circ}$, and $270^{\circ}$ fashion, just the same as that with the proposed single series-parallel sequential rotation feeding network.

Figure 3 shows the geometry of the 64-element antenna arrays. It can be seen that the 4-element antenna array with single series-parallel sequential rotation feeding network is used as a subarray to construct the larger arrays. Figure 3(a) shows the double applications of the sequential rotation technique: first to four patches to form a 4-element subarray, and then all the 4-element subarrays to form a 64-element array. Figure 3(b) shows the conventional antenna array with the conventional parallel feeding network.

To investigate the performance of the series-parallel sequential rotation feeding network further, we also designed two 256-element antenna arrays, as depicted in Figure 4. Figure 4(a) shows the antenna array with the sequential rotation technique applied twice. The reference antenna array, shown in Figure 4(b), uses the conventional parallel feeding technique. 


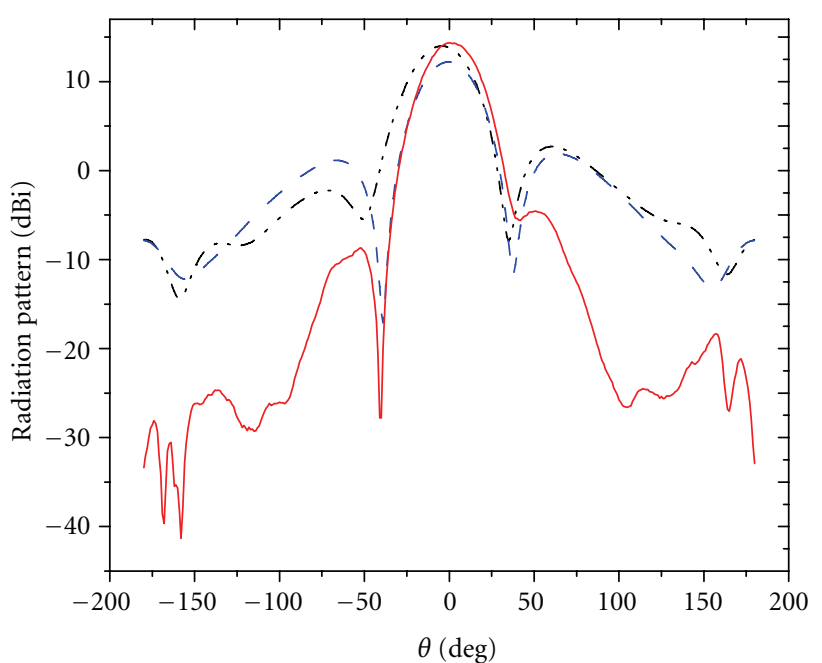

..... Simulated result of the proposed 4-element CP antenna array

— Measured result of the proposed 4-element CP antenna array

- - Simulated result of the conventional 4-element CP antenna array

(a)

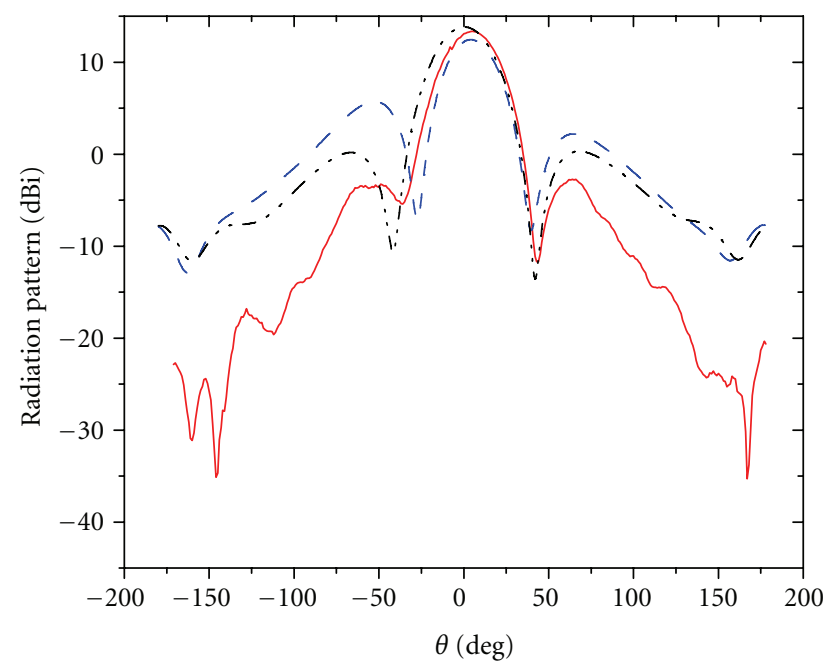

..... Simulated result of the proposed 4-element CP antenna array

Measured result of the proposed 4-element CP antenna array

- - Simulated result of the conventional 4-element $\mathrm{CP}$ antenna array

(b)

FIgURE 10: Measured and simulated radiation patterns of the 4-element CP antenna arrays at $29 \mathrm{GHz}$ : (a) $\varphi=0^{\circ},(\mathrm{b}) \varphi=90^{\circ}$.

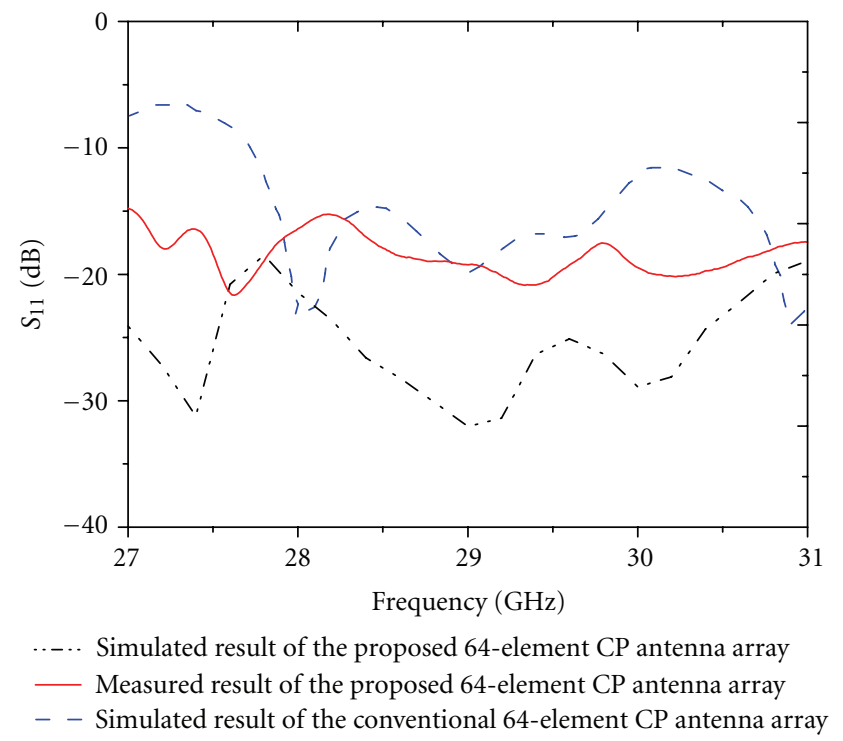

FIgURE 11: Measured and simulated $S_{11}$ of the 64-element CP antenna arrays.

\section{Simulated and Measured Results}

After simulation and optimization with Ansoft Designer and HFSS, the 4-element and 64-element CP antenna arrays with the parallel-series sequential rotation feeding networks are fabricated and tested. Their photographs are shown in Figures 5 and 6 . The measurement results are shown in Figures 7 and 8, along with the simulated results. For the 256-element CP antenna arrays, only simulated results of the arrays with parallel-series sequential rotation feeding networks and with parallel feeding networks are compared.
3.1. 4-Element CP Antenna Arrays. As seen from Figure 7, the measured and simulated results of the 4-element CP antenna array with the single series-parallel sequential rotation feeding network corroborate well for $S_{11}$. The measured bandwidth of the fabricated 4-element $\mathrm{CP}$ antenna array is about $4.33 \mathrm{GHz}$, or $14.75 \%$, from 27.19 to $31.52 \mathrm{GHz}$ [11]. The simulated result of the conventional 4-element $\mathrm{CP}$ antenna array with the parallel feeding network shows the bandwidth of about $3.0 \mathrm{GHz}$, or $10.34 \%$, from 27.25 to $30.25 \mathrm{GHz}$.

Figure 8 shows the measured and simulated axial ratio. The figure shows the fabricated 4-element $\mathrm{CP}$ antenna array 


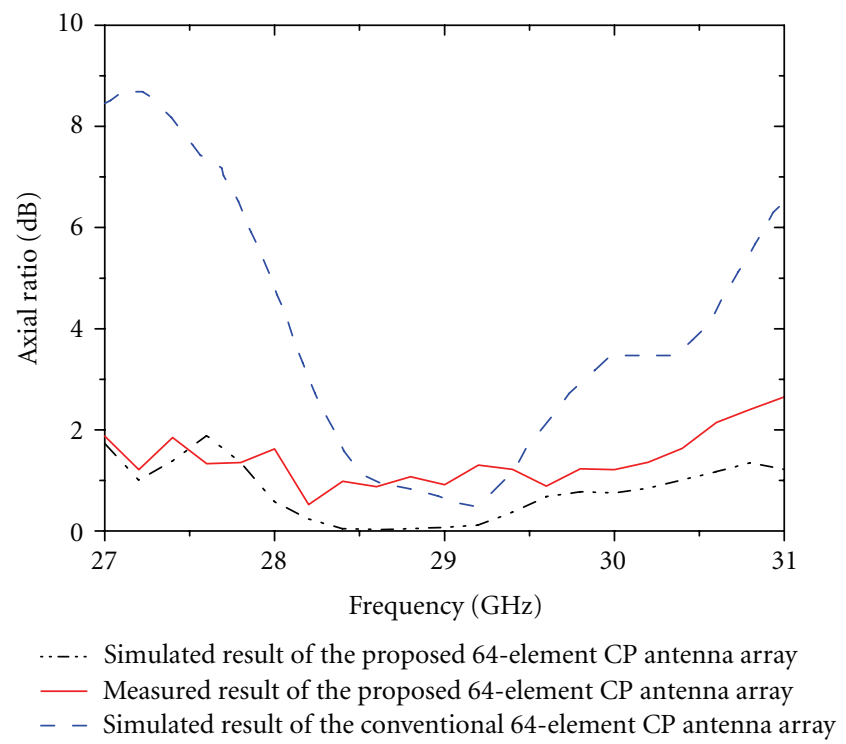

Figure 12: Measured and simulated axial ratio of the 64-element CP antenna arrays.

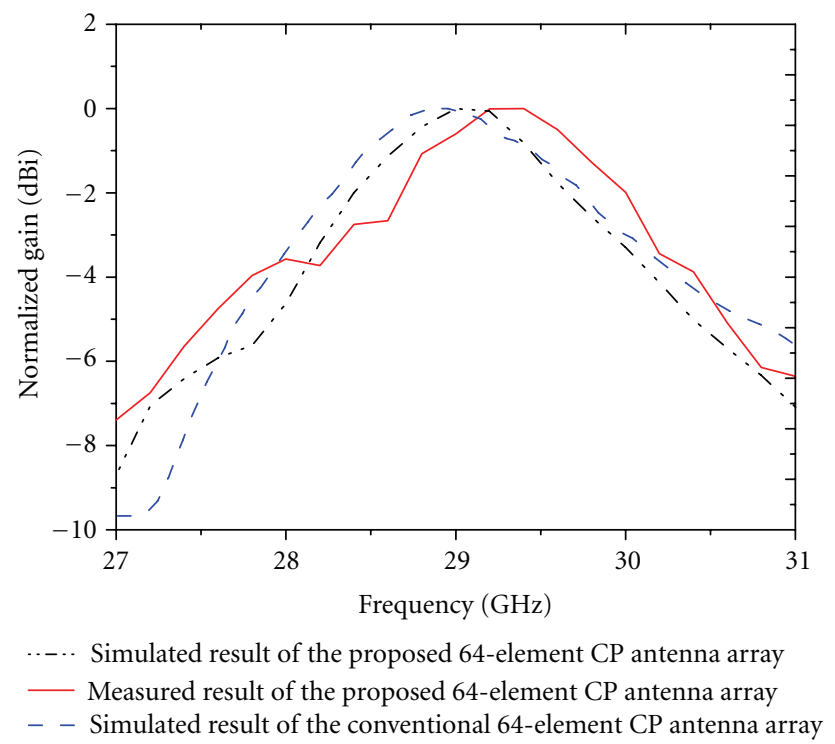

FIGURE 13: Measured and simulated gain of the 64-element CP antenna arrays.

has a 3 -dB bandwidth from $28.45 \mathrm{GHz}$ to over $30 \mathrm{GHz}$ [11], and the conventional 4-element $\mathrm{CP}$ antenna array's 3-dB bandwidth is from $28 \mathrm{GHz}$ to $29.61 \mathrm{GHz}$.

Figures 9 and 10 shows the measured and simulated gain and radiation patterns at $29 \mathrm{GHz}$, respectively. It can be seen that the measured sidelobes of the fabricated 4-element CP antenna array are lower than that of the simulated ones, and the gain of the conventional 4-element antenna array is about $1.5 \mathrm{dBi}$ lower than that of the proposed 4-element $\mathrm{CP}$ antenna array with the single series-parallel sequential rotation feeding network. This is mainly due to the fact that the parallel feeding network does not maintain the symmetry of the antenna array.
3.2. 64-Element CP Antenna Arrays. Figure 11 presents the measured and simulated reflection coefficients of the 64element CP antenna arrays with the double series-parallel sequential rotation feeding network and with the conventional parallel feeding network. It can be seen that the fabricated antenna array has a bandwidth from $27 \mathrm{GHz}$ to $31 \mathrm{GHz}$ [12]. Although the measured reflection coefficient of the proposed array is bigger than its simulated one, it is lower than the simulated coefficient of the conventional antenna array. Moreover, as seen from Figure 7, the double seriesparallel sequential rotation feeding network has the lower reflection coefficients than the conventional one; therefore, it reduces the RF power loss. 


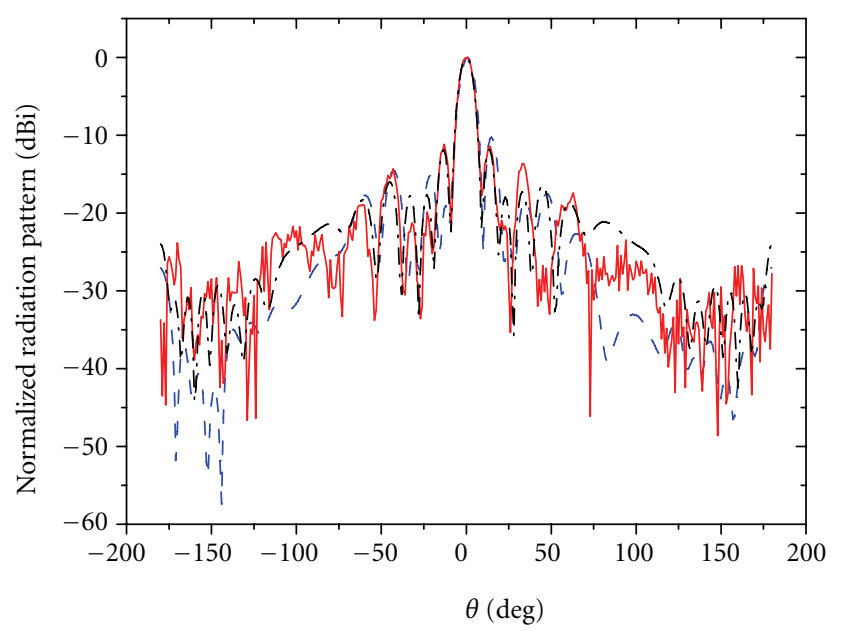

.... Simulated result of the proposed 64-element $\mathrm{CP}$ antenna array - Measured result of the proposed 64-element $\mathrm{CP}$ antenna array

- - Simulated result of the conventional 64-element $\mathrm{CP}$ antenna array

(a)

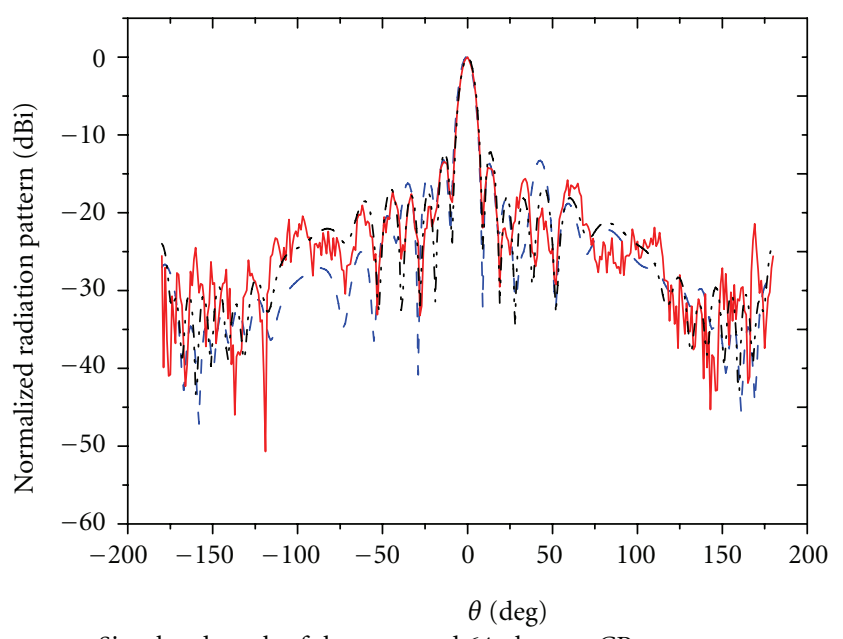

..... Simulated result of the proposed 64-element CP antenna array — Measured result of the proposed 64-element CP antenna array - - Simulated result of the conventional 64-element CP antenna array

(b)

Figure 14: Measured and simulated radiation patterns of the 64-element CP antenna arrays at $29 \mathrm{GHz}$ : (a) $\varphi=0^{\circ},(\mathrm{b}) \varphi=90^{\circ}$.

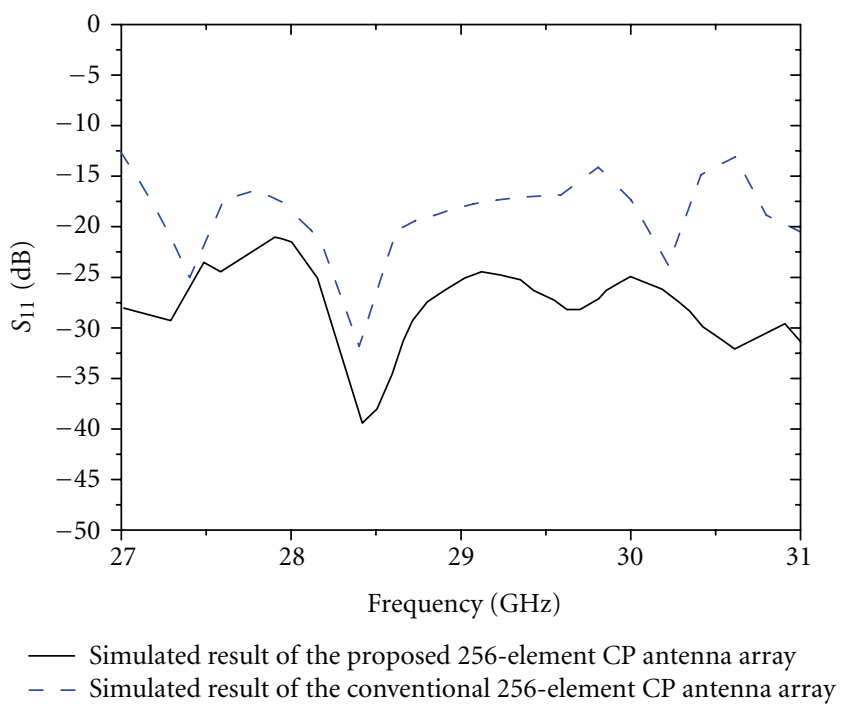

Figure 15: Simulated $S_{11}$ of the 256-element CP antenna arrays.

The measured and simulated axial ratio of the proposed 64-element CP antenna arrays is shown in Figure 12. The measured and simulated results are almost at the same level. The $3 \mathrm{~dB}$ axial ratio bandwidth covers from $27 \mathrm{GHz}$ to $31 \mathrm{GHz}[12]$. However, the conventional array has only the bandwidth of about $1.7 \mathrm{GHz}$, from $28.2-29.9 \mathrm{GHz}$.

Figures 13 and 14 represent the normalized peak gain versus frequency and the normalized radiation pattern at $29 \mathrm{GHz}$. The peak gain of the fabricated antenna array is found to be between 16 and $25 \mathrm{dBi}$ [12].

3.3. 256-Element CP Antenna Arrays. Figures 15, 16, 17 and 18 show the results with the 256-element CP antenna array in the frequency range from 27 to $31 \mathrm{GHz}$. The reflection coefficient is less than $-20 \mathrm{~dB}$, the axial ratio is less than $0.5 \mathrm{~dB}$ at most frequencies, and the peak gain is found to be between 20 and $30 \mathrm{dBi}$ (unfortunately, the authors are not allowed to disclose the exact values due to the proprietary requirement by the industrial sponsor of the work). All these results are better than that of the conventional array with the parallel feeding network; this once again proves that the series-parallel sequential rotation feeding network can improve the performance of the CP microstrip antenna arrays, especially when be used in large antenna arrays.

\section{Conclusion}

In this paper, we investigate the performance of $\mathrm{Ka}$-band $\mathrm{CP}$ microstrip antenna arrays with single, double and multiple 


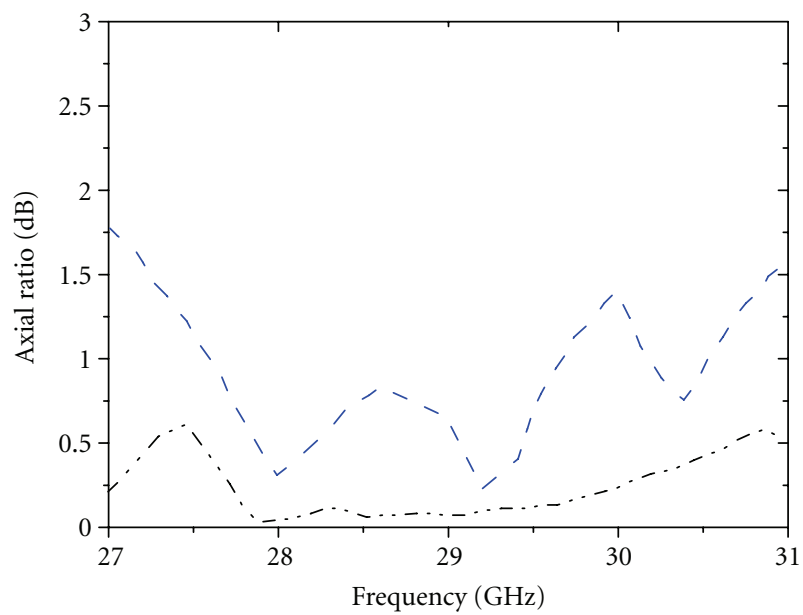

-... Simulated result of the proposed 256-element CP antenna array

- - Simulated result of the conventional 256-element CP antenna array

FIGURE 16: Simulated axial ratio of the 256-element CP antenna arrays.

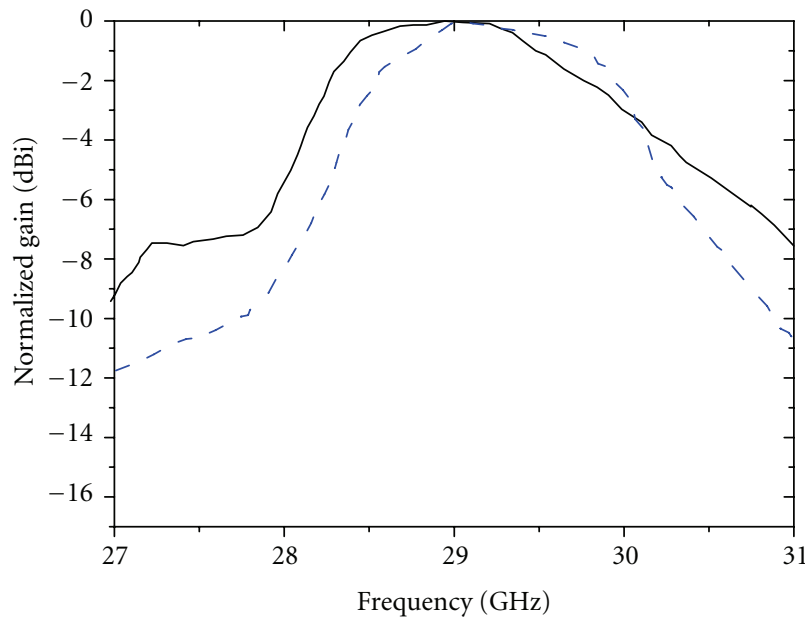

_ Simulated result of the proposed 256-element CP antenna array

- - Simulated result of the conventional 256-element CP antenna array

Figure 17: Simulated gain of the 256-element CP antenna arrays.

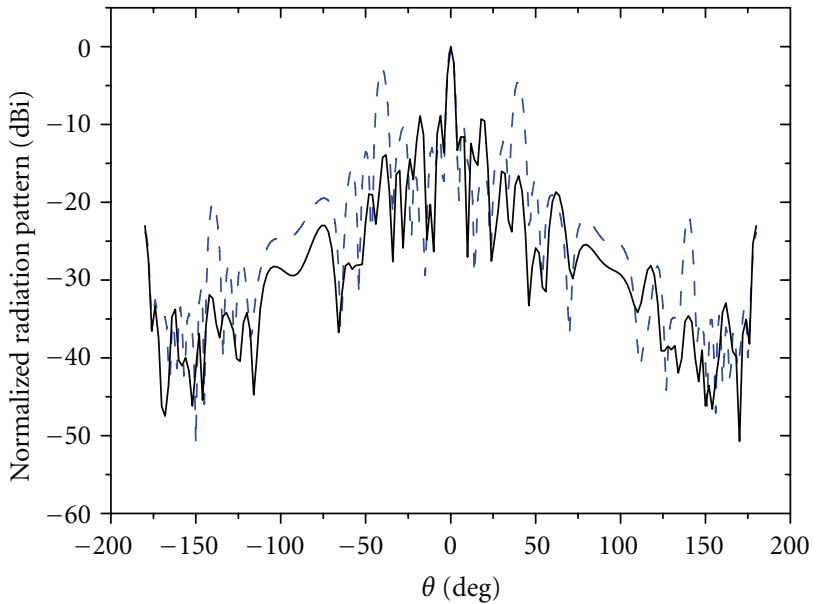

- Simulated result of the proposed 256-element CP antenna array

- - Simulated result of the conventional 256-element CP antenna array

(a)

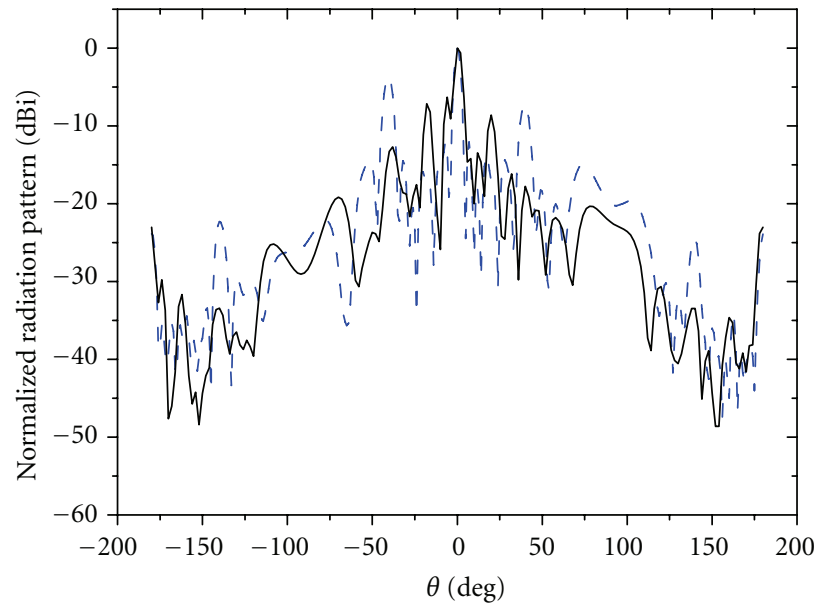

- Simulated result of the proposed 256-element CP antenna array - - Simulated result of the conventional 256-element CP antenna array

Figure 18: Simulated radiation patterns of the 256-element CP antenna arrays at $29 \mathrm{GHz}$ : (a) $\varphi=0^{\circ}$, (b) $\varphi=90^{\circ}$. 
series-parallel sequential rotation feeding network. The 4element, 64-element and 256-element CP antenna arrays are designed, simulated, fabricated, and tested. By comparing with the performance of the CP antenna arrays with the conventional parallel feeding network, it can be concluded that the multilevel series-parallel sequential rotation feeding technique can significantly reduce the RF loss, broaden the bandwidth, improve the $\mathrm{CP}$ purity, and achieve high gain. And the larger the arrays, the better the feeding networks perform. This feeding technique can be widely used in the design of antenna arrays in radar and satellite communication systems.

\section{Acknowledgment}

This work was supported in part by the National Science Foundation of China and NSAF under their joint Grant (no. 11076031).

\section{References}

[1] R. Garg, I. Bhartia, I. Bahl, and A. Ittipiboon, Microstrip Antenna Design Handbook, Artech House, Boston, Mass, USA, 2001.

[2] F. S. Chang, K. L. Wong, and T. W. Chiou, "Low-cost broadband circularly polarized patch antenna," IEEE Transactions on Antennas and Propagation, vol. 51, no. 10, pp. 3006-3009, 2003.

[3] R. Caso, A. Buffi, M. Rodriguez Pino, P. Nepa, and G. Manara, "A novel dual-feed slot-coupling feeding technique for circularly polarized patch arrays," IEEE Antennas and Wireless Propagation Letters, vol. 9, pp. 183-186, 2010.

[4] J. Huang, "Ka-band circularly polarized high-gain microstrip array antenna," IEEE Transactions on Antennas and Propagation, vol. 43, no. 1, pp. 113-116, 1995.

[5] B. Biglarbegian, M. Fakharzadeh, D. Busuioc, M. R. NezhadAhmadi, and S. Safavi-Naeini, "Optimized microstrip antenna arrays for emerging millimeter-wave wireless applications," IEEE Transactions on Antennas and Propagation, vol. 59, no. 5, pp. 1742-1747, 2011.

[6] M. Yousefbeigi, A. Enayati, M. Shahabadi, and D. Busuioc, "Parallel-series feed network with improved G/T performance for high-gain microstrip antenna arrays," Electronics Letters, vol. 44, no. 3, pp. 180-182, 2008.

[7] H. Legay and L. Shafai, "A self-matching wideband feed network for micro strip arrays," IEEE Transactions on Antennas and Propagation, vol. 45, no. 4, pp. 715-722, 1997.

[8] H. Evans, P. Gale, B. Aljibouri, E. G. Lim, E. Korolkeiwicz, and A. Sambell, "Application of simulated annealing to design of serial feed sequentially rotated $2 \times 2$ antenna array," Electronics Letters, vol. 36, no. 24, pp. 1987-1988, 2000.

[9] T. Yuan, N. Yuan, and L. W. Li, "A novel series-fed taper antenna array design," IEEE Antennas and Wireless Propagation Letters, vol. 7, pp. 362-365, 2008.

[10] E. Levine, G. Malamud, S. Shtrikman, and D. Treves, "Study of microstrip array antennas with the feed network," IEEE Transactions on Antennas and Propagation, vol. 37, no. 4, pp. 426-434, 1992.

[11] A. Chen, Y. Zhang, Z. Chen, and S. Cao, "A Ka-band high-gain circularly polarized microstrip antenna array," IEEE Antennas and Wireless Propagation Letters, vol. 9, pp. 1115-1118, 2010.
[12] A. Chen, Y. Zhang, Z. Chen, and C. Yang, "Development of a Ka-band wideband circularly polarized 64-element microstrip antenna array with double application of the sequential rotation feeding technique," IEEE Antennas and Wireless Propagation Letters, vol. 10, pp. 1270-1273, 2011. 

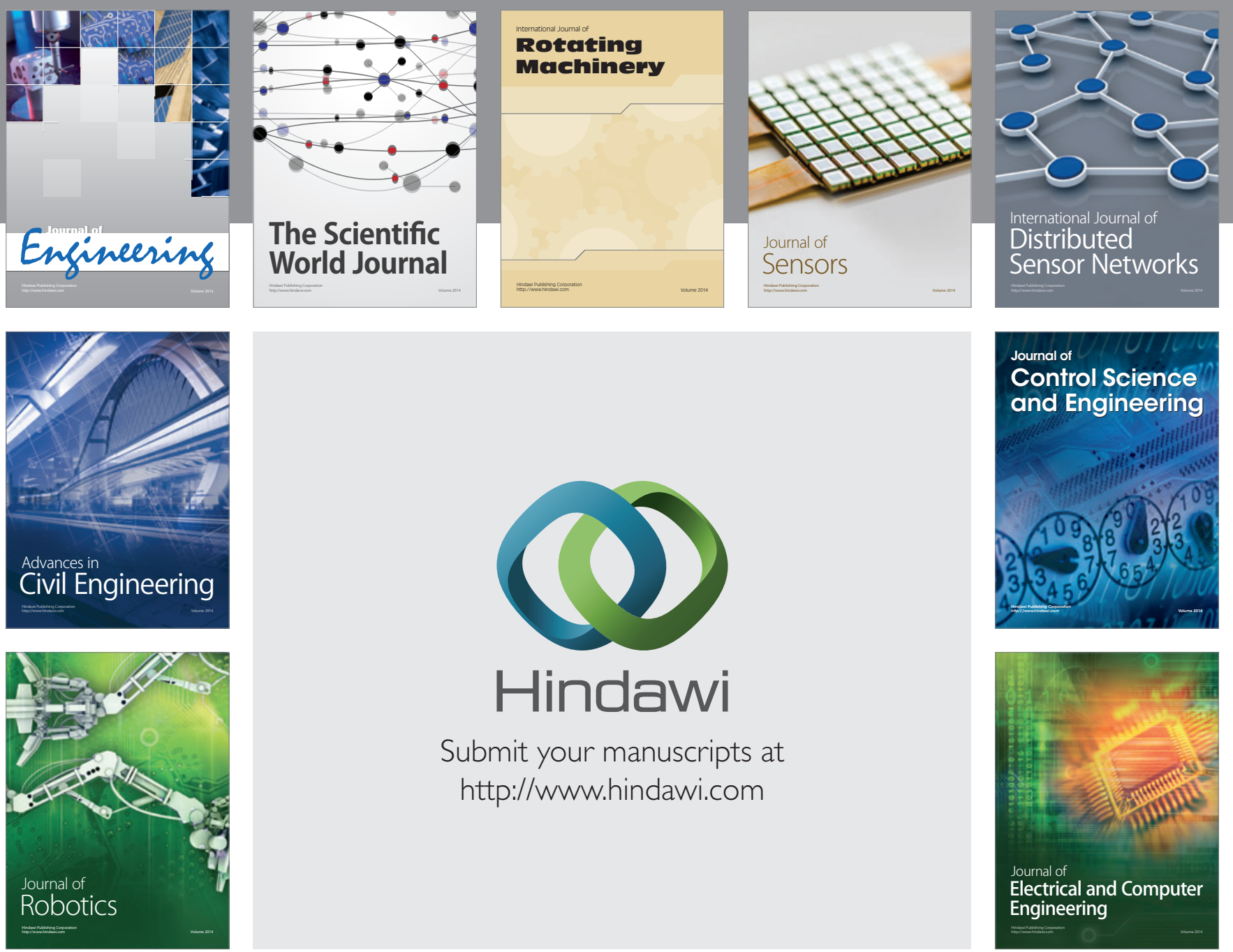

Submit your manuscripts at

http://www.hindawi.com
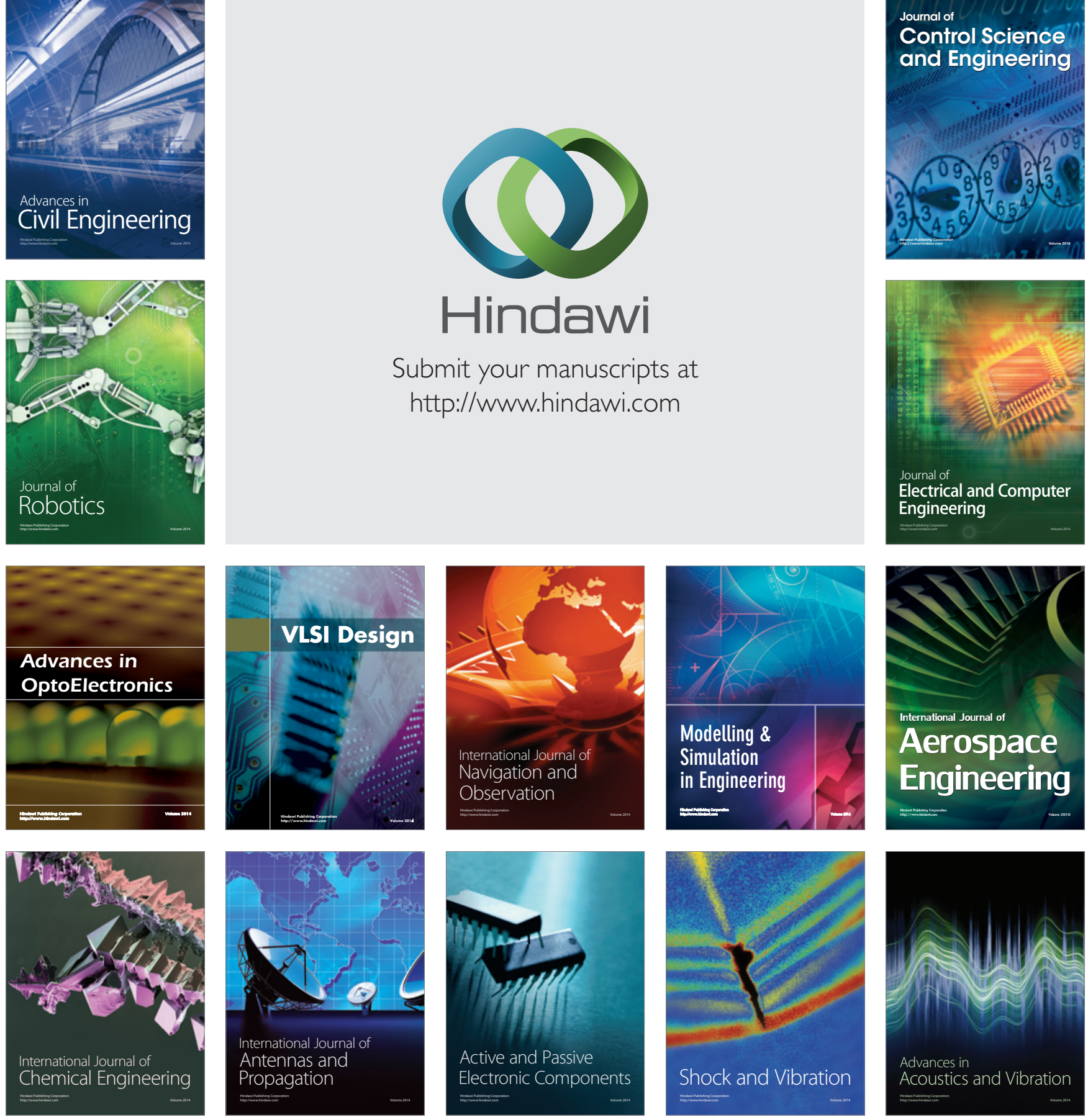